\title{
The Promise of Nanocomposite Thermoelectric Materials
}

\author{
M.S. Dresselhaus ${ }^{1}$, G. Chen $^{1}$, Z.F. Ren ${ }^{2}$, K. McEnaney ${ }^{1}$, G. Dresselhaus ${ }^{1}$, and J.-P. Fleurial ${ }^{3}$ \\ ${ }^{1}$ Massachusetts Institute of Technology, 77 Massachusetts Ave., Cambridge, MA 02139 U.S.A. \\ ${ }^{2}$ Boston College, 140 Commonwealth Ave., Chestnut Hill, MA 02467 USA \\ ${ }^{3}$ Jet Propulsion Laboratory, 4800 Oak Grove Dr., Pasadena, CA 91109 USA
}

\begin{abstract}
The concept of using nanocomposite thermoelectric materials in bulk form for practical applications is presented. Laboratory studies have shown the possibilities of nanostructures to yield large reductions in the thermal conductivity while at the same time increasing the power factor. Theoretical studies have suggested that structural ordering in nano-systems is not necessary for the enhancement of ZT, leading to the idea of using nanocomposites as a practical scale-up technology for making bulk thermoelectric materials with enhanced ZT values. Specific examples are presented of nanocomposite thermoelectric materials developed by our group based on the familiar silicon germanium system, showing enhanced thermoelectric performance through nano-structuring.
\end{abstract}

\section{INTRODUCTION: THE ENERGY CHALLENGE}

\section{Thermo-electricity in the larger energy picture}

During the 21st century inhabitants on our planet expect to be making a transition from today's fossil fuel based economy to an energy economy that will be sustainable over the long term. The drivers for this transition to a sustainable energy scenario include the increase in global population, going from today's 6.5 billion people to 8.9 billion (see figure 1(a)) by 2050 . The last 30 years have not only witnessed an increasing energy demand per person, but people worldwide have aspired to a higher standard of living, resulting in a super-linear increase in energy demand over this time period (see figure 1(b)). Despite the large efforts in increasing energy efficiency and to some degree energy conservation, the increasing energy demands, especially from the developing world (see figure 1(b)), have in large part contributed to the super-linear aspect of the increasing energy demand worldwide. Extrapolating into the future, the energy demands are expected to double from today's $15 \pm 2 \mathrm{TW}$ level to about the $30 \mathrm{TW}$ level by mid-century. To address this huge increase in energy demand over a short time period of 40 years is a daunting challenge to our global population, not only from an energy standpoint, but also from an environmental standpoint as over the last thousand years there has been a notable increase in global both mean temperature (by $\sim 1^{\circ} \mathrm{C}$ ) and $\mathrm{CO}_{2}$ levels (to about $380 \mathrm{PPM}$ ). 

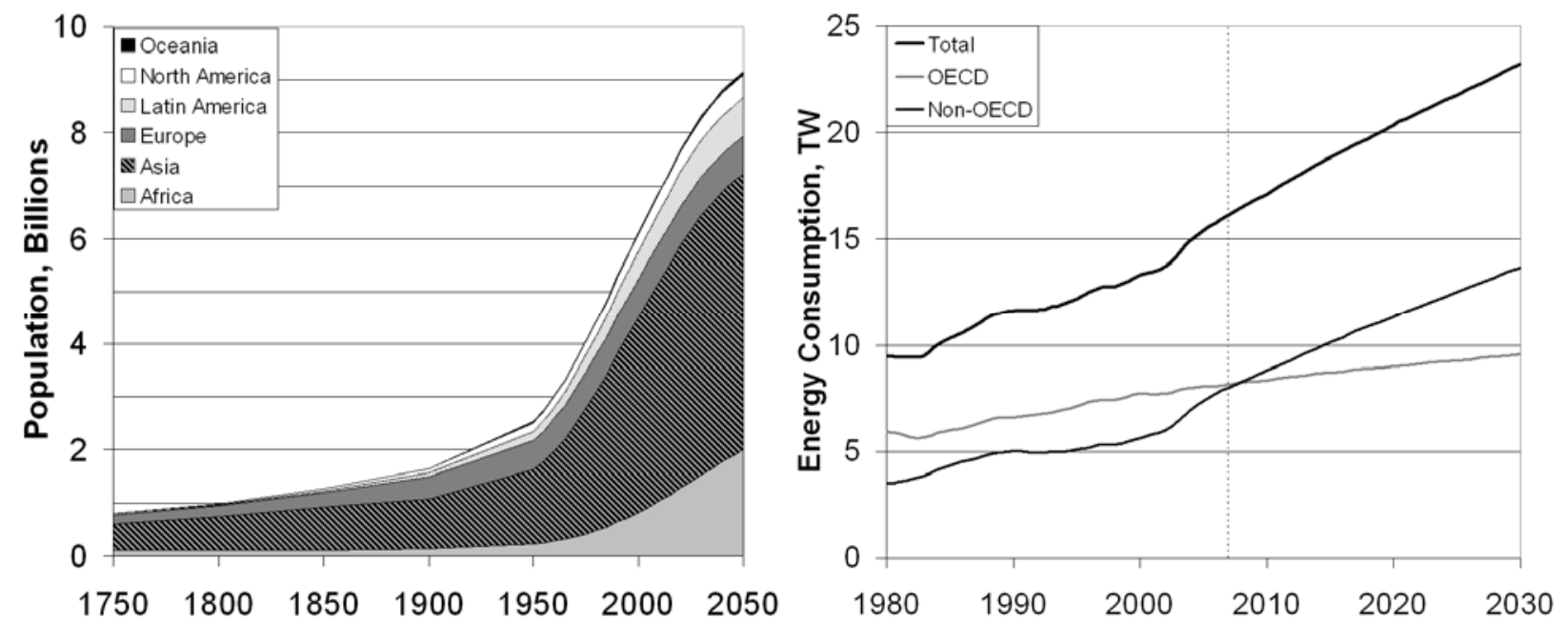

Figure 1. Historical and projected global population ${ }^{1,2}$ and energy demand ${ }^{3}$.

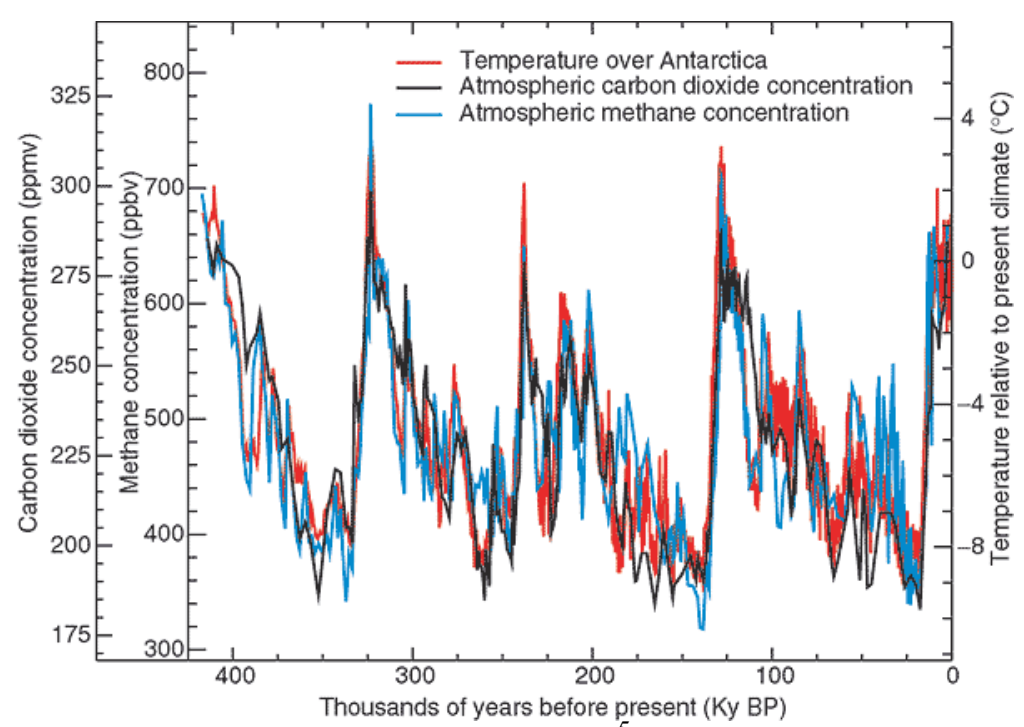

Figure 2. Ice core data ${ }^{5}$ for temperature and atmospheric $\mathrm{CO}_{2}$ and $\mathrm{CH}_{4}$.

When looking at data from ice core records that go back in history, we see that natural events on our planet (occurring over a period of $\sim 400,000$ years) result in fluctuations in the temperature of about $10^{\circ} \mathrm{C}$ over 100,000 year periods, so that the man-made effect of a $1^{\circ} \mathrm{C}$ rise in mean global temperature is modest. Predictions of an increase in temperature of about $2-3^{\circ} \mathrm{C}$ for the 21 st century is also not so large on the scale of figure 2. However, considering that this increase comes at a time when the earth is at a maximum mean temperature has serious implications on our sea level and climatic conditions. Even more threatening is the fact that the present global $\mathrm{CO}_{2}$ level is $380 \mathrm{ppm}$, which can be seen from figure 2 to be significantly higher than at any time in the past 400,000 years. Getting far out of equilibrium with the known atmospheric ranges that can be tolerated by planet earth is threatening and is having a strong effect on global strategies to increase efforts to greatly lower $\mathrm{CO}_{2}$ emissions. For example: many European Union nations have pledged to be on $20 \%$ renewable energy sources by the year $2020 .^{5}$ 
Figure 3 shows the implications of the transition to a sustainable energy scenario from today's fossil fuel economy where $85 \%$ of today's energy comes from oil, coal and gas with only a few percent coming from renewable sources, such as hydroelectric, wind and photovoltaics. The magnitude of the energy challenge involves both the huge amount of new energy supply that must be found and the short time scale over which this transition to a sustainable energy environment must take place. To achieve such a transition, major advances in basic research, engineering know-how and industrial development will be necessary, along with both energy conservation and better utilization of waste heat for electricity generation, two areas where thermoelectrics can play a major role. To make this happen, higher thermoelectric figure of merit, $Z T$, materials will be needed, as discussed further in this review.
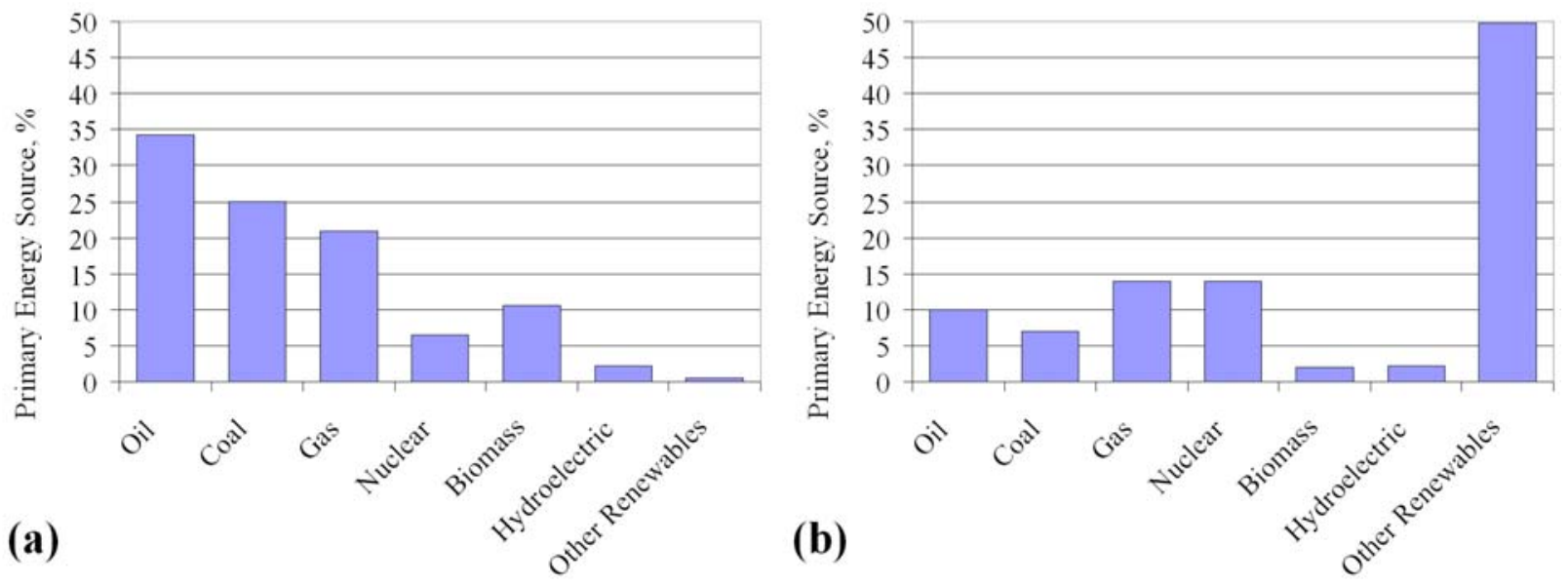

Figure 3. World primary energy consumption (a) $\operatorname{today}^{6}$ (2003) and (b) 2050 (target).

\section{THE THERMOELECTRIC MATERIALS CHALLENGE}

\section{Nanostructured materials for energy applications}

Nanostructured materials are important for energy-based applications overall. When materials have nano-sized features, quantum limits drive the physics, greatly expanding the parameter space for materials properties beyond what can be obtained in conventional materials. Nanostructured materials also can have orders of magnitude more surface area per unit volume, thus promoting catalytic interactions and surface interactions. Since catalysis involves providing energy to overcome an energy barrier, thereby introducing an exponential factor, catalysis allows large benefits to be realized in reaction product for modest increases in thermal energy. Such large scale approaches for change are especially helpful in addressing the daunting scales in magnitude and time of the energy challenge. In addition, engineering materials on the nanoscale can allow independent control of parameters that are interdependent in bulk materials; this aspect of nanomaterials is utilized especially for the case of thermoelectric materials.

\section{$\underline{\text { An introduction to thermoelectrics }}$}

Thermoelectric devices are based on the Seebeck effect and allow for the generation of electrical energy directly from a supply of thermal energy. The Seebeck effect occurs when a 
material is subjected to a temperature gradient, where electrons diffuse from the hotter region of the material towards the colder region and consequently build up a small voltage - on the order of micro volts per ${ }^{\circ} \mathrm{C}$ temperature difference between the two ends of the material. If dissimilar p- and n-type materials are connected electrically in series and thermally in parallel a more substantial voltage can be developed, which can then be used to supply electrical power to a load. The voltage obtained is directly proportional to the temperature difference that can be maintained: $\Delta \mathrm{V}=\mathrm{S} \Delta \mathrm{T}$. The overall performance depends on three key material properties that affect the device's ability to sustain the temperature difference during operation when current is flowing. Improving thermoelectric efficiency hinges on: increasing the Seebeck coefficient $S$, so that larger voltages can be obtained for each leg of the thermoelectric device; decreasing the thermal conductivity $\kappa$ as to minimize the heat being conducted (parasitic heat leakage) through the leg, which warms the cold side and decreases the temperature difference; and increasing the electrical conductivity $\sigma$, so that joule heating is minimized (parasitic heat generation), which also serves to warm the cold side.

Thermoelectric materials have been used in a wide variety of applications. Radioisotope generators, which consist of a radioisotope core surrounded by thermoelectric materials coupled to a heat sink, have been used on NASA deep-space and long-life missions to supply power to satillites going to Jupiter and beyond. These thermoelectric devices are reliable and well-proven: the radioisotope generators on many spacecraft, including both Voyager missions launched in 1977, are still operating. Thermoelectrics can be used for earth-based power generation in place of AC generators at remote sites not connected to an electric grid. There is also growing interest in using thermoelectrics to recover waste heat, a potentially huge energy resource. By running the thermoelectric cycle in reverse, thermoelectrics can be used in cooling applications, and are especially effective when traditional working-fluid cooling cycles are not practical, such as miniature refrigerators, computer chip heat sinks, and cooled automobile seats. Thermoelectrics are also used as sensor elements for infrared detectors. The recent explosion in the production of cooled automobile seats has spurred a large expansion in the thermoelectric industry at a time when laboratory demonstrations of enhanced materials performance have been carried out in many laboratories. Therefore the stage is set for an increase in interest in thermoelectric materials containing nanostructures. In this review we discuss examples of major advances that have occurred with the development of SiGe nanocomposite systems and conclude with comments on the potential impact of these research developments on the future directions for the field.

\section{Nanostructured thermoelectric materials}

Thermoelectrics are heat engines, limited by the second law of thermodynamics. The efficiency of a thermoelectric generator is related to the dimensionless figure of merit, $Z T$, which is the product of the temperature, the electrical conductivity, and the square of the Seebeck coefficient, divided by the thermal conductivity: $Z T=T \sigma S^{2} / \kappa$. In traditional bulk materials, $S, \sigma$, and $\kappa$ are functions of the carrier concentration of the material and cannot be controlled independently. In low-dimensional and other advanced materials, the connection between these parameters can be broken: distorting the density of states can increase the Seebeck coefficient without decreasing the electrical conductivity, and increasing the likelihood of an interface scattering event at a grain boundary that can reduce the thermal conductivity more than the 
electrical conductivity. Both of these effects have been shown to increase the thermoelectric figure of merit dramatically.

\section{State-of-the-art thermoelectric materials}

The current research focus in advancing thermoelectric materials performance is in four areas: phonon glass/electron crystals (PGECs), low-dimensional materials, nanostructured materials, and materials with resonant peaks in the conduction band density of states near the Fermi level, as given in the following examples. PGEC materials such as skutterudites contain atoms which can rattle inside "cages" within the crystal structure; these atoms scatter phonons but not electrons, thus reducing the lattice thermal conductivity without strongly affecting the electrical conductivity. Researchers ${ }^{7}$ have measured a $Z T$ of nearly 1.4 at $800 \mathrm{~K}$ in the skutterudite $\mathrm{Ba}_{\mathrm{x}} \mathrm{Yb}_{\mathrm{y}} \mathrm{Co}_{4} \mathrm{Sb}_{12}$. Low-dimensional materials rely on quantum effects of very small length scales to affect the electronic density of states, and to scatter phonons preferentially to electrons. Two-dimensional $\mathrm{Bi}_{2} \mathrm{Te}_{3} / \mathrm{Sb}_{2} \mathrm{Te}_{3}$ super-lattices ${ }^{8}$ have exhibited a $Z T$ of 2.4 at $300 \mathrm{~K}$. $Z T$ values of 1.6 at $300 \mathrm{~K}$ have been achieved in zero-dimensional $\mathrm{PbSe}_{0.98} \mathrm{Te}_{0.02} / \mathrm{PbTe}$ quantum dots $^{9}$. Nanostructured materials, such as BiSbTe that has been ball-milled and hot-pressed, also have exhibited ${ }^{10}$ a $Z T$ near 1.4 at about $373 \mathrm{~K}$. Thallium-doped PbTe, a bulk material with a resonant peak in the density of states, has shown ${ }^{11}$ a $Z T$ of 1.5 at $773 \mathrm{~K}$. The next phase of the thermoelectrics materials industry seems to require a large expansion of usage. This will require the production of large quantities of thermoelectric modules, and requiring more large-scale production approaches. Here nanocomposite thermoelectric materials are expected to play a special role, once the next generation of materials are better developed.

\section{RECENT ADVANCES IN THERMOELECTRIC MATERIALS SCIENCE AND TECHNOLOGY}

\section{Overview of nanocomposites}

A promising approach to achieving large quantities of bulk materials with a high ZT is the creation of bulk materials with nano-sized features. One of these approaches is in development of nanocomposite materials for thermoelectric applications. By synthesizing materials with very small grains, phonon scattering can be greatly enhanced, with only a small reduction in electrical conductivity. Production of these materials can be rapidly ramped up because they can be synthesized in large batches.

Bulk materials are the starting components for our nanocomposites. These bulk materials can be based on pure elements, or complex alloys, such as skutterudites. Chunks of these starting materials are mixed with dopants and ball-milled until the mean grain size and grain size distribution is on the appropriate scale for a particular application. For example, ball-milling compounds for a few hours may result in a 20 -nm average particle size, but contains a large distribution of subgrains at the 20 -nm scale. ${ }^{10}$ After the constituents have been mixed and reduced to nanometer size in the ball mill, the resulting powder of nanoparticles is rapidly pressed into ingots with either a DC press or a hot press so that the increase in the grain size distribution is small.

In order to improve $Z T$, the lattice thermal conductivity must be decreased. Recent modeling has provided insight into the effect of grain orientation and size on phonon transport 
(see figure 4), and shows that it is the amount of interface area in a sample that scatters phonons more than electrons that dominates the thermal conductivity. ${ }^{12}$ It has been shown that in silicon at room temperature, $80 \%$ of the lattice thermal conductivity comes from phonons with mean free paths less than $10 \mu \mathrm{m}$. Nanostructures restricting the phonon mean free path to $10 \mathrm{~nm}$ could reduce the lattice thermal conductivity by nearly two orders of magnitude ${ }^{13}$ (as can be seen in figure 5).

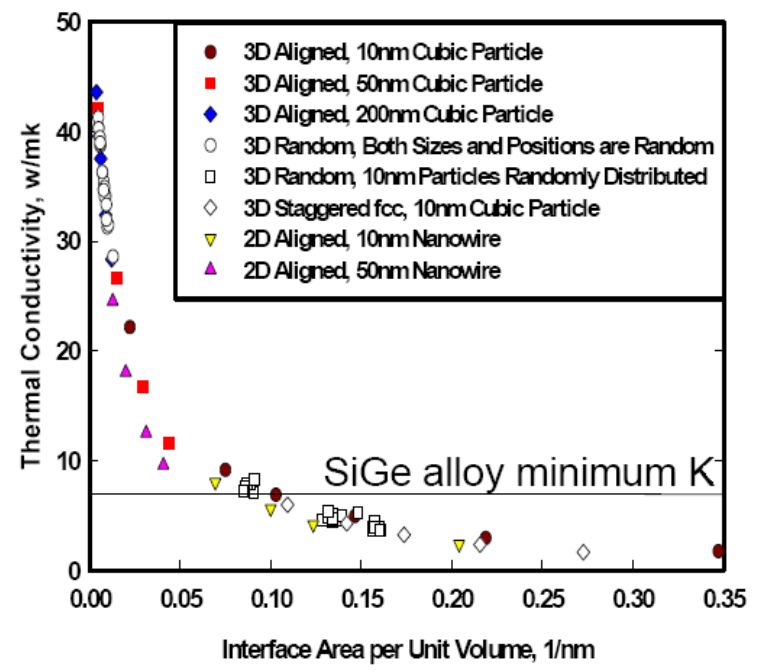

Figure 4. Plot of the thermal conductivity vs. interface area per unit volume shows a universal behavior. $^{12}$
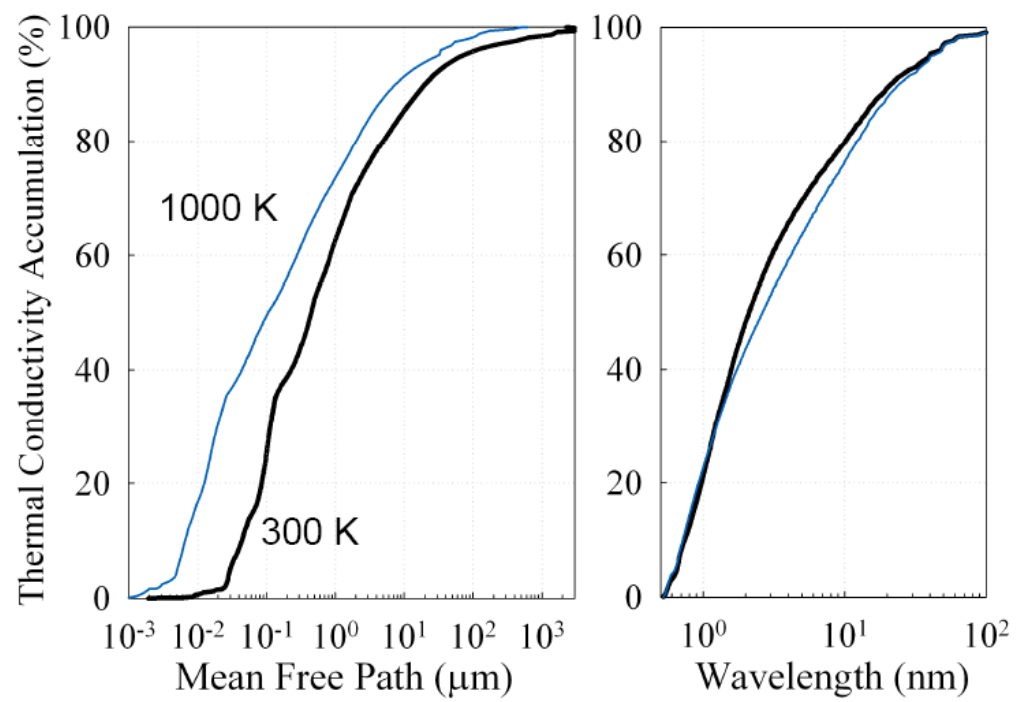

Figure 5. Thermal conductivity accumulation as a function of mean free path (left) and wavelength (right) at two different temperatures. ${ }^{13}$

Modeling of nanocomposite thermoelectrics has shown that there are large temperature ranges over which the power factor can increase and the thermal conductivity can decrease at the same time. ${ }^{14}$ Experimental studies of the thermal conductivity and power factor comparing their temperature-dependent performance in 3D bulk materials with our nanocomposite material of the same composition show that these desirable effects only occur in nano-scale materials (see figure 
6). Here the power factor and the thermal conductivity for a $\mathrm{Si}_{0.80} \mathrm{Ge}_{0.20}$ nanocomposite thermoelectric material are compared to the corresponding bulk sample used to power NASA space missions, showing superior thermoelectric performance of nanocomposite $\mathrm{Si}_{0.80} \mathrm{Ge}_{0.20}$, with characteristics not found in 3D materials. ${ }^{15}$ In fact, gains in $Z T$ up to $40 \%$ have been made in the $\mathrm{Bi}_{2} \mathrm{Te}_{3}$ system simply by decreasing the grain size. ${ }^{10}$ In these demonstrations of enhanced $Z T$ it is also important to include measurements of devices which exhibit enhanced thermoelectric performance as was done in this case, thereby confirming the findings from the physical properties measurements. Boundaries between nano-sized grains restrict the phonon mean free path through boundary scattering, thereby reducing the lattice contribution to the thermal conductivity. The power factor increase for the nanocomposites largely comes from an increase in carrier density.
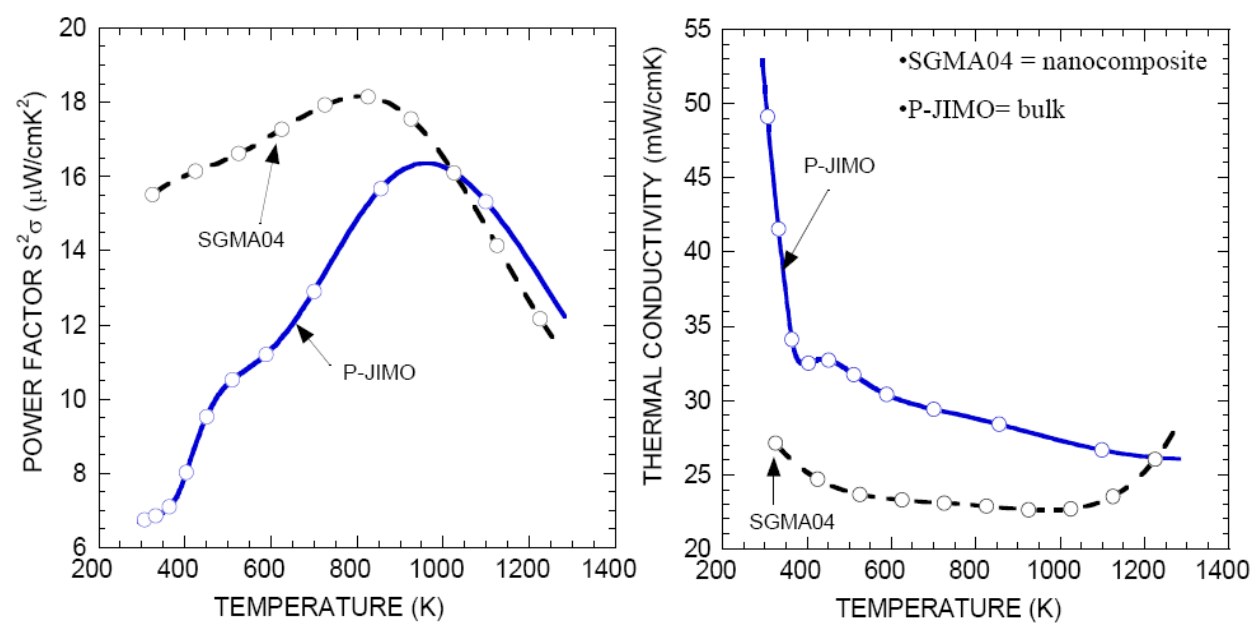

Figure 6. Comparison of thermoelectric properties (power factor on the left and thermal conductivity on the right) for bulk vs. nanostructured materials based on work done at JPL. ${ }^{15}$

\section{RECENT RESULTS ON Si ${ }_{1-\mathrm{x}} \mathrm{Ge}_{\mathrm{x}}$ NANOCOMPOSITES}

In this section we report very promising recent results on the thermoelectric performance of $\mathrm{Si}_{1-\mathrm{x}} \mathrm{Ge}_{\mathrm{x}}$ nanocomposite sytems as an example of a nanocomposite version of a materials system that is now in use by NASA for thermoelectric applications for power generation on space satellites. Bulk $\mathrm{Si}_{1-\mathrm{x}} \mathrm{Ge}_{\mathrm{x}}$ is a good high-temperature thermoelectric material, with a $Z T$ approaching 1 at $1200 \mathrm{~K}$. Both silicon and germanium are commonly used in the semiconductor industry. Silicon is a plentiful material and the grade of material needed for thermoelectric applications is relatively inexpensive and non-toxic. $\mathrm{Si}_{1-\mathrm{x}} \mathrm{Ge}_{\mathrm{x}}$ can easily be doped with donors (n-type) or acceptors (p-type). Both $\mathrm{n}$ - and $\mathrm{p}$-type materials benefit from nanostructuring and are stable up to at least $1050 \mathrm{C}$. Values of $Z T=1$ for $\mathrm{p}$-type $\mathrm{Si}_{0.80} \mathrm{Ge}_{0.20}$ and $Z T=1.3$ for $\mathrm{n}$-type $\mathrm{Si}_{0.80} \mathrm{Ge}_{0.20}$ nanocomposite materials have been reported ${ }^{16,17}$ with substantial enhancement over the performance of their bulk 3D counterparts. The reason for the more than $50 \%$ increase in $Z T$ for the p-type $\mathrm{Si}_{0.80} \mathrm{Ge}_{0.20}$ doped with boron is associated with the increased phonon scattering at the grain boundaries. This arises from the small grains in the powder material produced by the ball milling process, as shown in figure 7, where we see from the x-ray diffraction pattern that the powder is a single-phase material with a mean particle size of $15 \mathrm{~nm}$. TEM images showing particles of a wide range of sizes with many in the $\sim 20 \mathrm{~nm}$ size range and most of the particles 
contain many much smaller multigrains within the large grains. Furthermore, the compaction process to produce the nanocomposite material shows no significant increase in grain size. The very large distribution of grain sizes in figure 7 is highly effective in increasing the scattering of the wide distribution of phonons present in this material, thereby reducing the thermal conductivity by a factor of about 2 , as seen in figure 8 where results for $\sigma(T), S(T), \kappa(T)$, and $Z T(T)$ are shown for the nanocomposite material relative to the bulk material with the same $\mathrm{Si}_{0.80} \mathrm{Ge}_{0.20}$ ratio. By achieving clean boundaries between grains and the full mass density of $\mathrm{Si}_{0.80} \mathrm{Ge}_{0.20}$ in the nanocomposite material, the high power factor of the bulk material is reproduced in the nanocomposite. The $Z T$ thus achieved with the nanocomposite material is about $90 \%$ higher than that of the bulk p-type $\mathrm{Si}_{0.80} \mathrm{Ge}_{0.20}$ material now flown for NASA missions. The stability of the nanostructure at elevated temperatures (with tests done at $1100^{\circ} \mathrm{C}$ for 7 days) was investigated by annealing studies at elevated temperatures and the results on the thermoelectric property measurements are shown by the open circles in figure 8 .

a

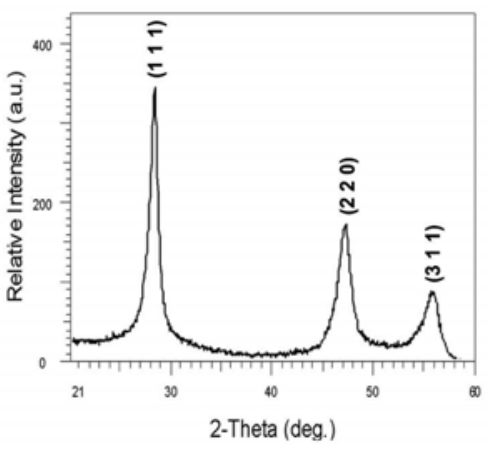

C

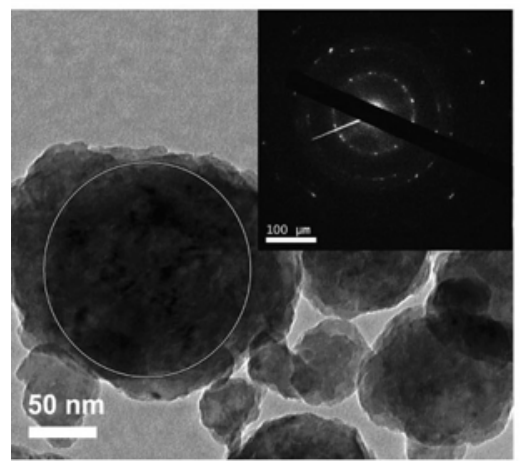

b

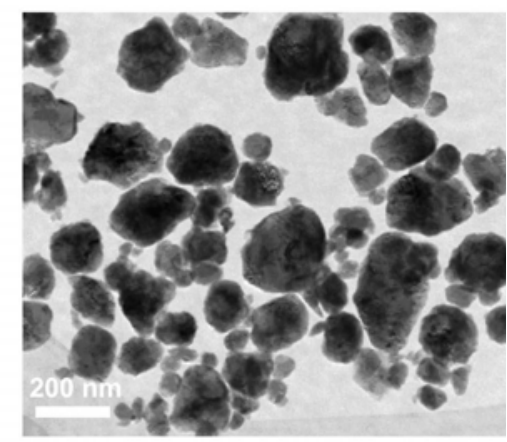

d

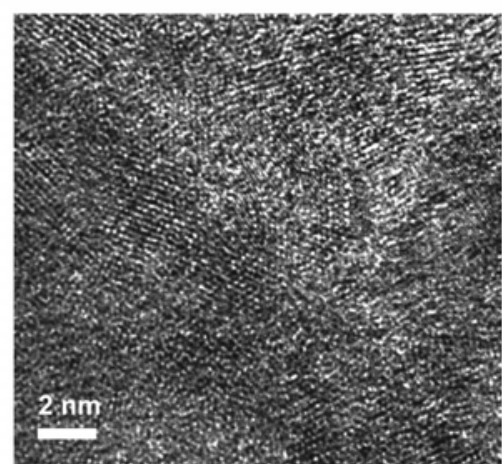

Figure 7. (a) XRD and (b),(c),(d) low, medium, and high-resolution TEM characterization studies on p-type $\mathrm{Si}_{0.80} \mathrm{Ge}_{0.20}$. . Inset shows electron diffraction measurements, indicating a collection of crystallites within a single grain. 

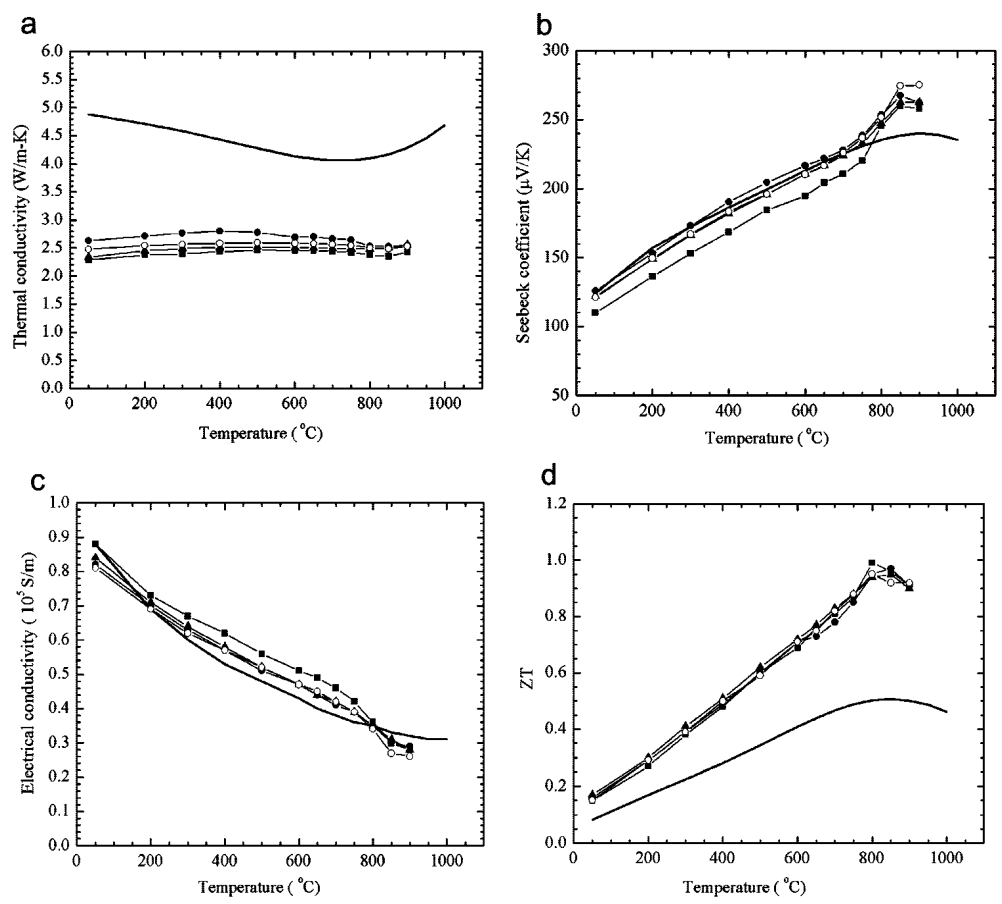

Figure 8. Temperature dependent (a) $\kappa(T)$, (b) $S(T)$, (c) $\sigma(T)$, and (d) $Z T(T)$ measurements of ptype nanocomposite $\mathrm{Si}_{0.80} \mathrm{Ge}_{0.20}$ material compared to $3 \mathrm{D}$ bulk material of the same composition (see text). ${ }^{16}$

Similar success was achieved with $\mathrm{n}$-doped $\mathrm{Si}_{0.80} \mathrm{Ge}_{0.20}$ material doped with phosphorus ${ }^{17}$ as shown in figure 9 for which a $Z T \sim 1.3$ was obtained. In this case, a similar scenario to that for the p-type $\mathrm{Si}_{0.80} \mathrm{Ge}_{0.20}$ nanocomposite material was achieved, with a major reduction in $\kappa$ while maintaining or slightly increasing the power factor, and maintaining good thermal stability under elevated temperature $\left(1050^{\circ} \mathrm{C}\right.$ in this case) operation.
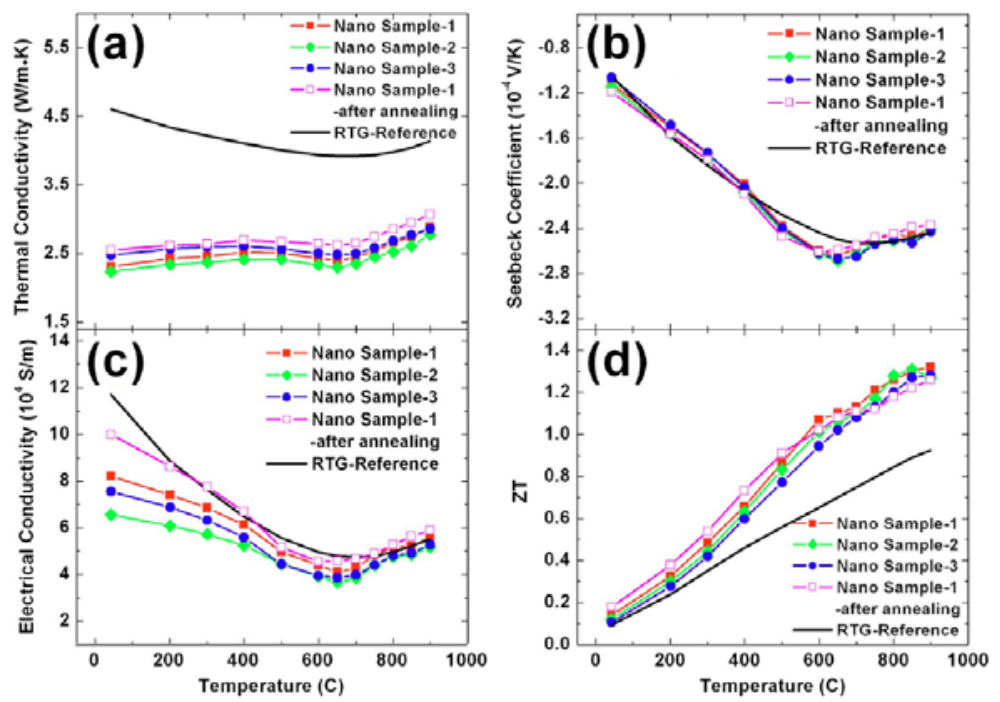

Figure 9. Temperature dependent (a) $\kappa(T)$, (b) $S(T)$, (c) $\sigma(T)$, and (d) $Z T(T)$ measurements of ntype nanocomposite $\mathrm{Si}_{0.80} \mathrm{Ge}_{0.20}$ samples compared to $3 \mathrm{D}$ bulk material of the same composition flown by NASA. ${ }^{17}$ 
Since $\mathrm{Ge}$ is more expensive and less abundant than $\mathrm{Si}$, there is motivation toward reducing the amount of Ge used in the $\mathrm{Si}_{1-\mathrm{x}} \mathrm{Ge}_{\mathrm{x}}$ alloy. Therefore scientific studies to determine the Ge concentration for the optimization of $Z T$ led to studies of nanocomposites with low $\mathrm{Ge}$ concentration, namely $\mathrm{Si}_{0.95} \mathrm{Ge}_{0.05}$ alloys ${ }^{18}$. Results for the thermoelectric performance shown in figure 10 suggest that $5 \%$ Ge is sufficient to make a major reduction in $\kappa(T)$ and to yield an increase in $Z T$ that is comparable to that for the $\mathrm{Si}_{0.80} \mathrm{Ge}_{0.20}$ bulk thermoelectric material now flown by NASA. Modeling calculations clearly show that alloying strongly reduces the mean free path for phonons with wavelength shorter than $\sim 4 \mathrm{~nm}$ due to scattering from point defects and grains of small size. The nanograined material is effective at scattering over the extended range of phonon wavelengths of interest for the $\mathrm{Si}_{1-\mathrm{x}} \mathrm{Ge}_{\mathrm{x}}$ system. The lower Ge concentration,on the other hand, results in a higher carrier mobility. Thus, by varying the doping level and the grain size of nanostructures, some degree of control of the temperature variation of the various thermoelectric parameters can be achieved. This flexibility in materials preparation should prove useful for optimizing the performance of this high temperature thermoelectric system by varying the alloying ratio between $\mathrm{Si}$ and $\mathrm{Ge}$.
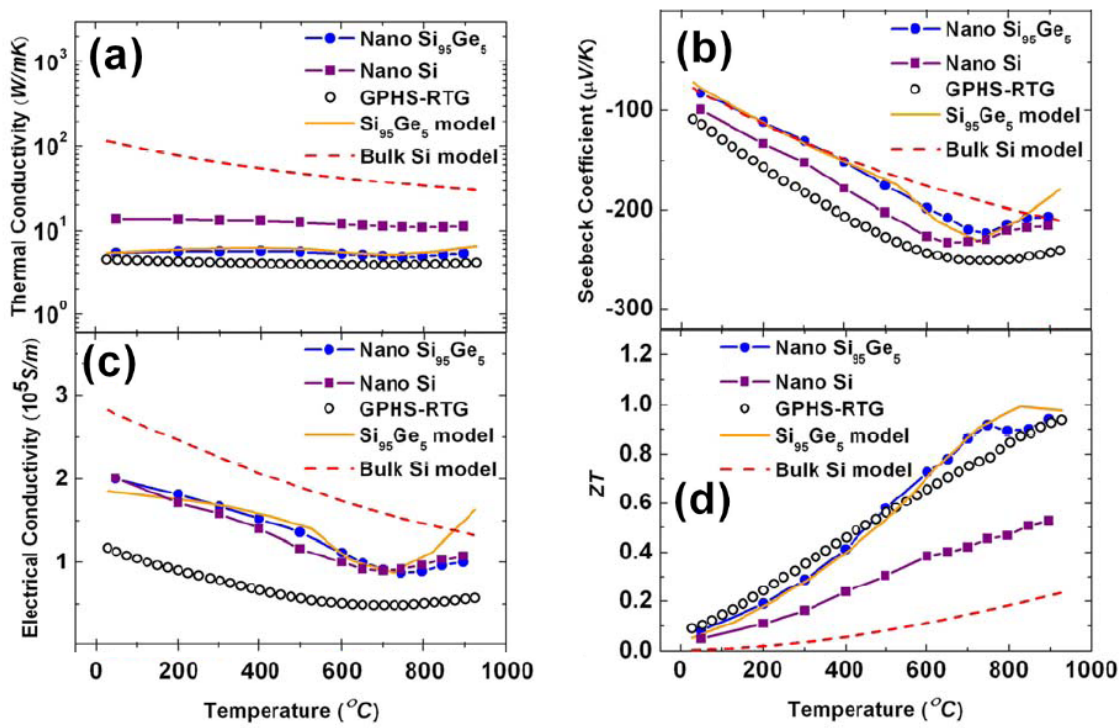

Figure 10. Temperature dependent (a) $\kappa(T)$, (b) $S(T)$, (c) $\sigma(T)$, and (d) $Z T(T)$ measurements of bulk nanocomposite $\mathrm{Si}_{0.95} \mathrm{Ge}_{0.05}$ samples in comparison to those on $\mathrm{Si}_{0.80} \mathrm{Ge}_{0.20}$ and $\mathrm{Si}^{18}$

\section{OUTLOOK TO THE FUTURE}

\section{Capturing waste heat with thermoelectrics}

Until now, thermoelectrics have focused on niche applications where their long-term reliability was exploited. With the increased efficiency now coming on line and the increasing global focus on energy conservation and waste heat recovery, we can foresee more widespread use of thermoelectricity in addressing new aspects of the energy challenge. Thermoelectric materials offer tremendous opportunity to convert waste heat into electricity. We can foresee an increasing emphasis given to such uses in the future. As humans burn fuel in automobiles, power plants, manufacturing processes, and buildings, the majority of the fuel's energy is wasted 
as it passes out tailpipes, up chimneys, or through walls and windows. In each of these situations, a significant amount of the waste heat could be converted by thermoelectrics into useful electricity. Automotive engineers are now working on replacing the alternator in automobiles with thermoelectric devices in the exhaust stream ${ }^{19}$. These thermoelectric devices could intercept $65 \%$ of a vehicle's fuel energy now going into the coolant and exhaust streams, thereby resulting in an increase in the fuel efficiency. In residential buildings, thermoelectrics could be implemented in the heating systems to effect a cogeneration system, with the electricity supplementing or ideally replacing the power from the electrical grid. Waste heat from manufacturing processes presently produce an enormous energy stream that has yet to be tapped. Frequently, the waste stream is at a very high temperature, giving high-quality heat that yields a high maximum potential (Carnot) efficiency and should be an attractive target for energy conservation. The increased efficiency of the newly-developed nanocomposite thermoelectric materials described in this review are likely to make such application areas more attractive.

However, as we look into the future, we are even more likely to look for totally new applications of thermoelectric materials that we have not considered before. One such likely direction is a means of capturing solar energy, as discussed below.

\section{Solar power}

If we intend to live sustainably on this planet, all our energy must eventually come from renewable and sustainable resources. The sun provides four orders of magnitude more power than humans consume; tapping this resource will be the biggest challenge of the $21^{\text {st }}$ century. Our main tools for harnessing solar energy are photovoltaics, solar thermal systems, and thermoelectrics. The current rate of photovoltaic production and its expected acceleration in the coming years is not at a rate that will allow us to reach a goal of $1.5 \mathrm{TW}$ production by 2050 . Photovoltaic cell production would have to follow a trend similar to Moore's Law for semiconductors, with rates doubling every few years if we are to reach expected goals. Thus far, solar thermoelectrics have been largely neglected from widespread consideration, but they could become a key component in a renewable energy portfolio. The promise of solar thermoelectrics is that, unlike photovoltaics, they can use the entire solar spectrum, and they also can recover nearly all the energy of a photon, whereas photovoltaics can only obtain a bandgap of energy from a photon, no matter how energetic it is. Solar thermoelectrics do this more efficiently and at lower cost by focusing the sun's rays onto a selective surface which is black in the solar spectrum and white in the IR, which acts as the hot side for a thermoelectric element. A third technology that draws from both of these concepts is solar thermophotovoltaics, in which the sun's rays are again captured on a selective surface. This thermal energy is then reradiated from the back side of the selective surface onto a parallel photovoltaic cell. The back side of the selective surface is designed to emit at the optimal wavelength for the thermophotovoltaic, maximizing the efficiency of energy conversion from the sun into electricity. Designing these selective absorbers and emitters is challenging, but the payoff is an $85 \%$ theoretical maximum efficiency in absorbing the solar energy.

\section{Looking to the Future}

Recent advances in thermoelectric materials show promise for a wide range of applications. Laboratory tests have proven new techniques for increasing the thermoelectric figure of merit. 
As these results become validated, the thermoelectric field is gaining momentum and is growing. The next step is for researchers and industry to tackle the problems that are now preventing widespread implementation of thermoelectric devices. First, more fundamental studies must be carried out to better understand the physics driving thermoelectric properties. Second, efforts must be made to optimize promising thermoelectric materials systems. Third, industry must attempt to scale up promising materials and approaches in order to make low cost, safe products in large volumes. Finally, there must be appropriate attention given to the interplay between materials properties and system design to maximize total system efficiency. As we continue down this path of increasing efficiency and decreasing cost, new opportunities will present themselves as targets for thermoelectric applications, broadening the scope of the applicability of thermoelectrics in the larger energy landscape.

\section{ACKNOWLEDGMENTS}

The authors gratefully acknowledge the assistance of Mario Hofmann for help with the preparation of the figures. MSD, GC, and ZR acknowledge support for this work under DOE Grant DE-FG02-08ER46516. Part of this work was performed at the Jet Propulsion Laboratory, California Institute of Technology under contract with the National Aeronautics and Space Administration.

\section{REFERENCES}

1. Population data from 1950 - 2050: Population Division of the Department of Economic and Social Affairs of the United Nations Secretariat. World Population Prospects: The 2008 Revision. United Nations, 2008.

2. Population data from 1750 - 1950: United Nations. The World at Six Billion. United Nations, 1999.

3. U.S. Department of Energy. Energy Information Administration. Report\# DOE/EIA-0484: International Energy Outlook 2008. Washington: Government Printing Office (2008).

4. J.T. Houghton et al., Climate change 2001: The Scientific Basis. (IPCC, Cambridge, UK, 2001).

5. World International Renewable Energy Conference, Washington, 2008.

6. International Energy Agency. Energy to 2050: Scenarios for a Sustainable Future. (IEA, France 2003).

7. X. Shi et al., Appl. Phys. Lett. 92 182101, (2008).

8. R. Venkatasubramanian, E. Siivola, T. Colpitts, and B. O’Quinn, Nature 413 597-602 (2001).

9. T.C. Harman, P.J. Taylor, M.P. Walsh, and B.E. LaForge, Science 297 (5590) 2229-2232 (2002).

10. B. Poudel et al., Science 320 (5876) 634-638 (2008).

11. J.P. Heremans et al., Science 321 (5888) 554-557 (2008).

12. R. Yang, Ph.D. dissertation, Massachusetts Institute of Technology, 2006.

13. A.S. Henry and G. Chen, J. Comput. Theor. Nanosci., 5, 141-152 (2008).

14. A.J. Minnich, M.S. Dresselhaus, Z.F. Ren, and G.Chen, Energy Environ. Sci. (in press).

15. M.S. Dresselhaus et al., Advanced Materials, 19, 1043-1053 (2007).

16. G. Joshi et al., Nano Lett. 8 (12) 4670-4674 (2008).

17. X.W. Wang et al., Appl. Phys. Lett. 93193121 (2008). 
18. G. Zhu et al., Phys. Rev. Lett. (in press).

19. K. Matsubara, in $21^{\text {st }}$ International Conference on Thermoelectrics, Portland, OR, 2002, pp. 418-423. 